\title{
Mass corrections to the hard thermal or dense loops
}

\author{
Marc Comadran $\odot^{*}$ and Cristina Manuel $\odot^{\dagger}$ \\ Instituto de Ciencias del Espacio (ICE, CSIC), C. Can Magrans s.n., 08193 Cerdanyola del Vallès, \\ Catalonia, Spain and Institut d'Estudis Espacials de Catalunya (IEEC), \\ C. Gran Capità 2-4, Ed. Nexus, 08034 Barcelona, Spain
}

(Received 28 June 2021; accepted 14 September 2021; published 7 October 2021)

\begin{abstract}
We compute corrections to the hard thermal (or dense) loop photon polarization tensor associated to a small mass $m$ of the fermions of an electromagnetic plasma at high temperature $T$ (or chemical potential $\mu$ ). To this aim we use the on-shell effective field theory, amended with mass corrections. We also carry out the computation using transport theory, reaching to the same result. Intermediate steps in the computations reveal the presence of potential infrared divergencies. We use dimensional regularization, as it is respectful with the gauge symmetry, and then show that all infrared divergencies cancel in the final result. We compare the mass corrections with both the power and two-loop corrections, and claim that they are equally important if the mass is soft, that is, of order $e T$ (or $e \mu$ ), where $e$ is the gauge coupling constant, but are dominant if the mass obeys $e T<m \ll T$ (or $e \mu<m \ll \mu$ ).

DOI: 10.1103/PhysRevD.104.076006
\end{abstract}

\section{INTRODUCTION}

Relativistic QED and QCD plasmas have attracted the interest of the physics community for their wide range of applications in both cosmological, astrophysical and also heavy-ion physics. In their weak coupling regime perturbative computations of different physical observables require the resummation of Feynman diagrams [1,2], the so called hard thermal loops (HTL), to attain a result valid at a certain order in the gauge coupling expansion (see [3] for a review and complete set of basic references). This makes the studies of relativistic plasmas particularly cumbersome.

For very large values of the temperature $T$ (or of the chemical potential $\mu$ ), a well-defined hierarchy of energy scales appears in these relativistic plasmas, that allows for effective field theory descriptions, very similar to those applied for nonthermal physics. In a series of papers [4-9], the on-shell effective field theory (OSEFT) has been developed in order to describe the physics of the hard scales, or scales of order $T$ (or $\mu$ ), which are on-shell degrees of freedom. This effective field theory was initially developed to obtain quantum corrections to classical transport equations. Then it was realized that it could be used to improve the description of the hard scales of the plasma, and

\footnotetext{
marc.comadranc@e-campus.uab.cat

cmanuel@ice.csic.es
}

Published by the American Physical Society under the terms of the Creative Commons Attribution 4.0 International license. Further distribution of this work must maintain attribution to the author(s) and the published article's title, journal citation, and DOI. Funded by SCOAP ${ }^{3}$. as by-product, also the soft scales of order $e T$ (or $e \mu$ ), where $e$ is the gauge coupling constant.

The rationale and technical tools used by OSEFT are the same as that of other effective field theories, such as high density field theory (HDET) [10], or soft collinear effective field theory (SCET) [11,12], for example. After fixing the value of the high energy scale, in this case the energy of the (quasi) massless fermion, which is of order $\sim T$ for thermal plasmas, one defines some small fluctuations around that scale. Integrating out the high energy modes, one is left with an effective theory for the lower scales or quantum fluctuations. The resulting Lagrangian is organized as an expansion of operators of increasing dimension over powers of the high energy scale.

In this manuscript we focus our attention to thermal corrections to the HTL photon polarization tensor associated to the fact that fermions on the plasma might not be strictly massless, but have indeed a small mass $m$ much less than the temperature, $m \ll T$. This is a realistic assumption, as only in the cosmological epoch before the electroweak phase transition all elementary particles were strictly massless. While the power corrections to the HTL photon polarization tensor have been computed with OSEFT in Ref. [5], here we will use the OSEFT for the computation of the leading fermion mass corrections. We also check that the same result is obtained if derived from transport theory. Intermediate steps in the computations reveal the presence of potential infrared divergencies. A regularization of the momentum integrals is needed. We use dimensional regularization, as it is respectful with the gauge symmetry, and then show that all infrared divergencies cancel in the final result. We also 
explain why our results remain valid in the presence of a chemical potential, even for high values of $\mu$ and $T=0$.

This paper is structured as follows. In Sec. II we review the OSEFT Lagrangian including small mass corrections. We present the computation of the Feynman diagrams in OSEFT that provide mass corrections to the photon polarization tensor in Sec. III. The same result is obtained if computed from transport theory, as shown in Sec. IV. We then compare our results with both the power and two-loop corrections to the HTL in Sec. V, and discuss when the mass corrections are the dominant correction to the HTL polarization tensor. We denote with boldface letters 3 dimensional vectors. Natural units $\hbar=k_{B}=1$ are used throughout this manuscript.

\section{SMALL MASS CORRECTIONS TO THE OSEFT}

In this section we derive the OSEFT Lagrangian including mass corrections to the third order in the energy expansion. Let us briefly discuss how this is achieved.

In the spirit of the OSEFT we split the momentum of the energetic fermion as

$$
q^{\mu}=p v^{\mu}+k^{\mu},
$$

where $v^{\mu}$ is a lightlike vector, $p$ is the high scale, while $k^{\mu}$ is the so called residual momentum, associated to the quantum fluctuation, and is such that $k^{\mu} \ll p$. For the antifermion we will write

$$
q^{\mu}=-p \tilde{v}^{\mu}+k^{\mu},
$$

where $\tilde{v}^{\mu}$ is also a lightlike vector. We will impose that

$$
u^{\mu}=\frac{v^{\mu}+\tilde{v}^{\mu}}{2}
$$

where $u^{\mu}$ is a frame vector, such that $u^{2}=1$, thus, it is timelike.

The OSEFT Lagrangian including small mass corrections has been derived in Ref. [9], and in an arbitrary frame it reads

$$
\begin{aligned}
\mathcal{L}_{p, v}= & \bar{\chi}_{v}(x)\left(i v \cdot D+i \not D_{\perp} \frac{1}{2 p+i \tilde{v} \cdot D} i \not D_{\perp}-m^{2} \frac{1}{2 p+i \tilde{v} \cdot D}\right) \frac{\tilde{p}}{2} \chi_{v}(x) \\
& -\bar{\chi}_{v}(x)\left(m\left[\frac{1}{2 p+i \tilde{v} \cdot D}, i \not D_{\perp}\right]\right) \frac{\not{p}}{2} \chi_{v}(x),
\end{aligned}
$$

for the particle field $\chi_{v}$, where $\not_{\perp}=P_{\perp}^{\mu \nu} \gamma_{\mu} D_{\nu}$, and the transverse projector is defined as $P_{\perp}^{\mu \nu}=g^{\mu \nu}-\frac{1}{2}$ $\left(v^{\mu} \tilde{v}^{\nu}+v^{\nu} \tilde{v}^{\mu}\right)$. For antiparticles, the Lagrangian can be obtained after replacing $p \leftrightarrow-p$ and $v^{\mu} \leftrightarrow \tilde{v}^{\mu}$. The Lagrangian has the same structure of that of SCET amended with small mass corrections $[13,14]$. Note that OSEFT and SCET are different theories, as the power counting is not the same (see [7] for a discussion on this point).

In writing the above Lagrangian, one assumes that the covariant derivatives, defined as $i D_{\mu}=i \partial_{\mu}+e A_{\mu}$, are soft, meaning that they are much less than the high energy scale, which here it is $p$. Equally, one assumes that the mass is such that $m \ll p$. The Lagrangian can then be now expanded using that $p$ is the hard scale of the problem.

For applications of plasma physics in thermal field theory, it is convenient to use the frame at rest with the plasma, thus $u^{\mu}=(1, \mathbf{0})$. Then one can replace $\frac{\mathscr{x}}{2}$ by $\not h=\gamma_{0}$ (recall that $\not \chi_{v}=0$ ). Expanding the Lagrangian on the high energy scale, one gets easily the first two terms in the energy expansion, which respect chirality

$$
\mathcal{L}_{p, v}^{(0)}=\bar{\chi}_{v}(i v \cdot D) \gamma^{0} \chi_{v}
$$

$$
\mathcal{L}_{p, v}^{(1)}=-\frac{1}{2 p} \bar{\chi}_{v}\left(D_{\perp}^{2}+m^{2}-\frac{e}{2} \sigma_{\perp}^{\mu \nu} F_{\mu \nu}\right) \gamma^{0} \chi_{v} .
$$

It is convenient to introduce local field redefinitions to eliminate the temporal derivative appearing at second order, as in Ref. [5]. These simplify the computations at higher orders. Thus, after the field redefinition

$$
\chi_{v} \rightarrow \chi_{v}^{\prime}=\left(1+\frac{\not D_{\perp}^{2}+m^{2}}{8 p^{2}}\right) \chi_{v}
$$

the Lagrangian at second order reads

$$
\begin{aligned}
\mathcal{L}_{p, v}^{(2)}= & \bar{\chi}_{v}^{\prime} \frac{1}{8 p^{2}}\left(\left[\not D_{\perp},\left[i \tilde{v} \cdot D, \not D_{\perp}\right]\right]\right. \\
& -\left\{\not D_{\perp}^{2}+m^{2}, i v \cdot D-i \tilde{v} \cdot D\right\} \\
& \left.+2 i e m \tilde{v}^{\mu} F_{\mu \alpha} \gamma_{\perp}^{\alpha}\right) \gamma^{0} \chi_{v}^{\prime} .
\end{aligned}
$$

Note that the term linear in the mass describes a breaking of chirality. 
We will also need the Lagrangian up to third order. To eliminate temporal derivatives at that order, we perform the local field redefinition

$$
\begin{aligned}
\chi_{v} \rightarrow \chi_{v}^{\prime \prime}= & \left(1-\frac{i}{8 p^{3}} \not D_{\perp}\left[\tilde{v} \cdot D, \not D_{\perp}\right]+\frac{i}{16 p^{3}}\left(\not D_{\perp}^{2}+m^{2}\right)(v \cdot D-\tilde{v} \cdot D)-\frac{i}{16 p^{3}}\left(\not D_{\perp}^{2}+m^{2}\right) \tilde{v} \cdot D\right. \\
& \left.+\frac{m}{8 p^{3}}\left[i \tilde{v} \cdot D, i \not D_{\perp}\right]\right) \chi_{v}^{\prime},
\end{aligned}
$$

so that the final Lagrangian reads

$$
\begin{aligned}
\mathcal{L}_{p, v}^{(3)}= & \frac{1}{8 p^{3}} \bar{\chi}_{v}^{\prime \prime}\left(\left(\not D_{\perp}^{2}+m^{2}\right)^{2}+\left[i \tilde{v} \cdot D, \not D_{\perp}\right]^{2}-(i v \cdot D-i \tilde{v} \cdot D)\left(\not D_{\perp}^{2}+m^{2}\right)(i v \cdot D-i \tilde{v} \cdot D)\right. \\
& +(i v \cdot D-i \tilde{v} \cdot D) \not D_{\perp}\left[i \tilde{v} \cdot D, \not D_{\perp}\right]-\left[i \tilde{v} \cdot D, \not D_{\perp}\right] \not D_{\perp}(i v \cdot D-i \tilde{v} \cdot D) \\
& \left.+m\left\{i v \cdot D-i \tilde{v} \cdot D,\left[i \tilde{v} \cdot D, i \not D_{\perp}\right]\right\}\right) \gamma^{0} \chi_{v}^{\prime \prime} .
\end{aligned}
$$

Please note that in the limit $m=0$ we recover the same Lagrangians derived in Ref. [5]. The pieces which are quadratic in the mass can be recovered from those of Ref. [5] simply by replacing $\not D_{\perp}^{2} \rightarrow \not D_{\perp}^{2}+m^{2}$. The linear terms in $m$, originating from the expansion of the last term in Eq. (4), describe the breaking of the chiral symmetry induced by the fermion mass.

We present here how the OSEFT fermion propagators are modified in the presence of a small mass. The particle/ antiparticle projectors in the frame at rest with the plasma are defined as $P_{v}=\frac{1}{2} \not \gamma \gamma_{0}$ and $P_{\tilde{v}}=\frac{1}{2} \tilde{b} \gamma_{0}$, respectively. We introduce chirality projectors

$$
P_{\chi}=\frac{1+\chi \gamma_{5}}{2}, \quad \chi= \pm
$$

The propagators for a fermion of chirality $\chi$ in the Keldysh formulation of the real time formalism of thermal field theory Ref. [15] read

$$
\begin{gathered}
S_{\chi}^{R / A}(k)=\frac{P_{\chi} P_{v} \gamma_{0}}{k_{0} \pm i \epsilon-f(\mathbf{k}, m)}, \\
S_{\chi}^{S}(k)=P_{\chi} P_{v} \gamma_{0}\left(-2 \pi i \delta\left(k_{0}-f(\mathbf{k}, m)\right)\right. \\
\left.\quad \times\left(1-2 n_{F}\left(p+k_{0}\right)\right)\right),
\end{gathered}
$$

where $n_{F}(x)=1 /(\exp |x| / T+1)$ is the Fermi-Dirac equilibrium distribution function. The function $f(\mathbf{k}, m)$ determines the dispersion law, and it is expanded also, we denote as $f^{(n)}(\mathbf{k}, m)$ the $n$ order term in the $1 / p$ expansion. At lowest order

$$
f^{(0)}(\mathbf{k}, m)=k_{\|},
$$

and we have defined $k_{\|}=\mathbf{k} \cdot \mathbf{v}$, while

$$
\begin{aligned}
& f^{(1)}(\mathbf{k}, m)=k_{\|}+\frac{\mathbf{k}_{\perp}^{2}+m^{2}}{2 p}, \\
& f^{(2)}(\mathbf{k}, m)=k_{\|}+\frac{\mathbf{k}_{\perp}^{2}+m^{2}}{2 p}-\frac{k_{\|}\left(\mathbf{k}_{\perp}^{2}+m^{2}\right)}{2 p^{2}},
\end{aligned}
$$

as follows from Eqs. (6) and (8), respectively. The propagators for the antiparticle quantum fluctuations can be also easily deduced. They read

$$
\begin{gathered}
\tilde{S}_{\chi}^{R / A}(k)=\frac{P_{\chi} P_{\tilde{\imath}} \gamma_{0}}{k_{0} \pm i \epsilon-\tilde{f}(\mathbf{k}, m)}, \\
\tilde{S}_{\chi}^{S}(k)=-P_{\chi} P_{\tilde{\nu} \gamma_{0}}\left(-2 \pi i \delta\left(k_{0}-\tilde{f}(\mathbf{k}, m)\right)\left(1-2 n_{F}\left(-p+k_{0}\right)\right)\right),
\end{gathered}
$$

where the function $\tilde{f}(\mathbf{k}, m)$ can be obtained from $f(\mathbf{k}, m)$, with the replacements $\mathbf{v} \rightarrow-\mathbf{v}$ and $p \rightarrow-p$. Note the extra minus sign in the symmetric antiparticle propagator, absent in its particle counterpart.

In summary, the OSEFT fermion propagators in this case can be deduced from those of the massless case simply by replacing $\mathbf{k}_{\perp}^{2} \rightarrow \mathbf{k}_{\perp}^{2}+m^{2}$ in the function that determines the dispersion relation at every order in the energy expansion.

Note that, for convenience, we keep the propagators above unexpanded in this section, as done in Ref. [5], but in the explicit computation of the different diagrams they are to be expanded in a $1 / p$ series.

\section{DIAGRAMMATIC COMPUTATION OF THE MASS CORRECTION TO THE RETARDED PHOTON POLARIZATION TENSOR}

In this section we compute the mass corrections to the retarded photon polarization tensor computed in OSEFT. Recall that there are two possible different topological diagrams that contribute to the computation, the bubble and the tadpole diagrams, see Fig. 1. The tadpole diagrams, 
(a)

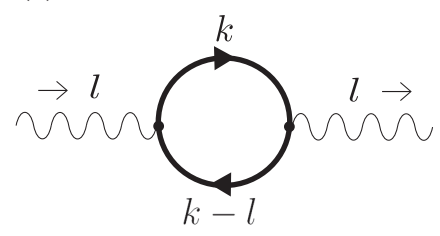

(b)

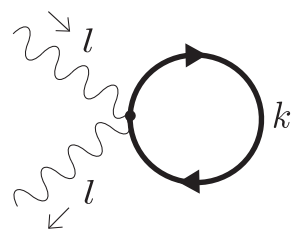

FIG. 1. (a) Bubble diagram (b) Tadpole diagram.

absent in QED, take into account particle-photon interactions mediated by an off-shell antiparticle (and viceversa for antiparticle-photon interactions), and are needed to respect the Ward identity obeyed by the polarization tensor, as we will explicitly check in this manuscript.

In the Keldysh representation the particle contribution to the bubble diagram of the retarded polarization tensor has the structure ${ }^{1}$

$$
\begin{aligned}
\Pi_{b, \chi}^{\mu \nu}(l)= & \frac{i}{2} \sum_{p, \mathbf{v}} \int \frac{d^{4} k}{(2 \pi)^{4}}\left\{\operatorname{Tr}\left[V^{\mu} S_{S}^{\chi}(k-l) V^{\nu} S_{R}^{\chi}(k)\right]\right. \\
& \left.+\operatorname{Tr}\left[V^{\mu} S_{A}^{\chi}(k-l) V^{\nu} S_{S}^{\chi}(k)\right]\right\},
\end{aligned}
$$

while the particle contribution to the tadpole diagram can be expressed as

$$
\Pi_{t, \mathcal{X}}^{\mu \nu}(l)=-\frac{i}{2} \sum_{p, \mathbf{v}} \int \frac{d^{4} k}{(2 \pi)^{4}} \operatorname{Tr}\left[W^{\mu \nu} S_{S}^{\chi}(k)\right],
$$

where the momentum dependence of the vertex functions $V^{\mu}$ and $W^{\mu \nu}$ are understood. Similar expressions can be written for the antiparticle contributions to the polarization tensor.

Using the explicit expressions of the fermion propagators, one can carry out the integral in $k_{0}$ to arrive to the general expressions

$$
\begin{aligned}
\Pi_{b, \chi}^{\mu \nu}(l)= & -\sum_{p, \mathbf{v}} \int \frac{d^{3} \mathbf{k}}{(2 \pi)^{3}} \operatorname{Tr}\left[V^{\mu} P_{\chi} P_{v} \gamma^{0} V^{\nu} P_{v} \gamma^{0}\right] \\
& \times \frac{n_{F}(p+f(\mathbf{k}-\boldsymbol{l}, m))-n_{F}(p+f(\mathbf{k}, m))}{l_{0}+i 0^{+}+f(\mathbf{k}-\boldsymbol{l}, m)-f(\mathbf{k}, m)},
\end{aligned}
$$

and

$$
\begin{aligned}
\Pi_{t, \chi}^{\mu \nu}(l)= & -\frac{1}{2} \sum_{p, \mathbf{v}} \int \frac{d^{3} \mathbf{k}}{(2 \pi)^{3}} \operatorname{Tr}\left[W^{\mu \nu} P_{\chi} P_{v} \gamma^{0}\right] \\
& \times\left(1-2 n_{F}(p+f(\mathbf{k}, m))\right),
\end{aligned}
$$

for the bubble and tadpole diagrams, respectively.

\footnotetext{
${ }^{1}$ We have changed the sign conventions of the definition of the polarization tensor as with respect to those used in Ref. [5].
}

The Feynman rules needed for the computation of the photon polarization tensor were given in Ref. [5] (see Tables I and II of that reference). In the presence of a mass in the OSEFT Lagrangian, new vertices appear proportional to the mass squared, which are given by

$$
\begin{gathered}
V_{(2), m^{2}}^{\mu}=-\frac{e m^{2}}{2 p^{2}} \gamma_{0} \delta^{\mu i} v^{i}, \\
W_{(3), m^{2}}^{\mu \nu}=-\frac{m^{2} e^{2} \gamma^{0}}{2 p^{3}}\left[P_{\perp}^{\mu \nu}+\frac{\left(v^{\mu}-\tilde{v}^{\mu}\right)\left(v^{\nu}-\tilde{v}^{\nu}\right)}{2}\right] .
\end{gathered}
$$

There are also new vertices proportional to the mass, which imply a change in the fermion chirality. At the order we will compute the mass corrections, $n=3$ in the energy expansion, these will not be needed, although they would be required at fourth order in the energy expansion. Note that at least two of these vertices would be needed in a computation of the photon polarization tensor to preserve the fermion chirality inside the loop.

We now evaluate the polarization tensor at different orders, noting that we either consider the energy expansion in the vertex functions, or in the fermion propagators, which can be used at the desired order of accuracy.

The first nonvanishing contribution to the photon polarization tensor occurs at $n=1$, but it does not carry any mass dependence. This was computed in Ref. [5], and it reproduces the HTL contribution. Let us recall the main results here. Adding the bubble and the tadpole diagrams at order $n=1$ gives

$\Pi^{\mu \nu}(l)=-e^{2} \sum_{\chi= \pm} \sum_{p, \mathbf{v}} \int \frac{d^{3} \mathbf{k}}{(2 \pi)^{3}} \frac{d n_{F}}{d p}\left(\frac{P_{\perp}^{\mu \nu}}{2}+v^{\mu} v^{\nu}-l_{0} \frac{v^{\mu} v^{\nu}}{v \cdot l}\right)$.

where the retarded prescription $l_{0} \rightarrow l_{0}+i 0^{+}$is understood.

It is now important to return to the original momentum variable $q^{\mu}$. Using the identity [5]

$$
\sum_{p, \mathbf{v}} \int \frac{d^{3} \mathbf{k}}{(2 \pi)^{3}} \equiv \int \frac{d^{3} \mathbf{q}}{(2 \pi)^{3}}
$$

and the relations [5]

$$
p=q-k_{\|, \hat{\mathbf{q}}}+\frac{\mathbf{k}_{\perp, \hat{\mathbf{q}}}^{2}}{2 q}+\mathcal{O}\left(\frac{1}{q^{2}}\right),
$$

$$
\mathbf{v}=\hat{\mathbf{q}}-\frac{\mathbf{k}_{\perp, \hat{\mathbf{q}}}}{q}-\frac{\hat{\mathbf{q}} \mathbf{k}_{\perp, \hat{\mathbf{q}}}^{2}+2 k_{\|, \hat{\mathbf{q}}} \mathbf{k}_{\perp, \hat{\mathbf{q}}}}{2 q^{2}}+\mathcal{O}\left(\frac{1}{q^{3}}\right),
$$




$$
\begin{aligned}
n_{F}(p)= & n_{F}(q)+\frac{d n_{f}}{d q}\left(-k_{\|, \hat{\mathbf{q}}}+\frac{\mathbf{k}_{\perp, \hat{\mathbf{q}}}^{2}}{2 q}\right) \\
& +\frac{1}{2} \frac{d^{2} n_{F}}{d q^{2}} k_{\|, \hat{\mathbf{q}}}^{2}+\mathcal{O}\left(\frac{1}{q^{3}}\right),
\end{aligned}
$$

where now the symbols $\mathbf{k}_{\|, \hat{\mathbf{q}}}$ and $\mathbf{k}_{\perp, \hat{\mathbf{q}}}$ denote the components of $\mathbf{k}$ parallel and perpendicular to $\hat{\mathbf{q}} \equiv \mathbf{q} / q$ with $q=|\mathbf{q}|$, respectively. We also define the vectors

$$
v_{\hat{\mathbf{q}}}^{\mu} \equiv(1, \hat{\mathbf{q}}), \quad \tilde{v}_{\hat{\mathbf{q}}}^{\mu} \equiv(1,-\hat{\mathbf{q}})
$$

After adding both the particle and antiparticle contributions to the photon polarization tensor one arrives to the well-known HTL expression

$$
\Pi_{\mathrm{htl}}^{\mu \nu}(l)=-4 e^{2} \int \frac{d^{3} \mathbf{q}}{(2 \pi)^{3}} \frac{d n_{F}}{d q}\left(\delta_{0}^{\mu} \delta_{0}^{\nu}-l_{0} \frac{v_{\hat{\mathbf{q}}}^{\mu} v_{\hat{\mathbf{q}}}^{\nu}}{v_{\hat{\mathbf{q}}} \cdot l}\right)
$$

At second order in the energy expansion, and in the absence of chiral misbalance, the Bose-Einstein statistics and the crossing symmetry demands that the polarization tensor $\Pi^{\mu \nu}(l)$ be symmetric under the simultaneous exchange of $\mu \leftrightarrow \nu$ and $l \leftrightarrow-l$ [16]. These symmetries explain the absence of linear terms in the photon momenta in the polarization tensor, which ultimately explain why there are not $n=2$ corrections in the polarization tensor in OSEFT. This was explicitly checked in Refs. [5,17]. This reasoning applies actually to all the even orders of the energy expansion, $n=4,6,8, \ldots$. We do not expect thus mass corrections at even orders, neither, and we actually have checked that there are none at $n=2$.

The first mass corrections to the photon polarization tensor occur at third order in the energy expansion, the same as the power corrections to the HTL computed in Refs. [5,17].

The mass dependent terms that arise in the bubble diagram are

$$
\begin{aligned}
\Pi_{b}^{\mu \nu}(l)= & -m^{2} e^{2} \sum_{\chi= \pm} \sum_{p, \mathbf{v}} \int \frac{d^{3} \mathbf{k}}{(2 \pi)^{3}}\left\{-\frac{l_{\|}}{2 p}\left[\left(\frac{d^{2} n_{F}}{d p^{2}}-\frac{1}{p} \frac{d n_{F}}{d p}\right) \frac{v^{\mu} v^{\nu}}{v \cdot l}-\frac{l_{\|}}{p} \frac{d n_{F}}{d p} \frac{v^{\mu} v^{\nu}}{(v \cdot l)^{2}}\right.\right. \\
& \left.\left.-\frac{1}{2 p} \frac{d n_{F}}{d p} \frac{v^{\mu}\left(v^{\nu}-\tilde{v}^{\nu}\right)+v^{\nu}\left(v^{\mu}-\tilde{v}^{\mu}\right)}{v \cdot l}\right]\right\},
\end{aligned}
$$

while in the tadpole one gets

$$
\Pi_{t}^{\mu \nu}(l)=-m^{2} e^{2} \sum_{\chi= \pm} \sum_{p, \mathbf{v}} \int \frac{d^{3} \mathbf{k}}{(2 \pi)^{3}}\left\{\frac{n_{F}}{2 p^{3}}\left[P_{\perp}^{\mu \nu}+\frac{\left(v^{\mu}-\tilde{v}^{\mu}\right)\left(v^{\nu}-\tilde{v}^{\nu}\right)}{2}\right]-\frac{P_{\perp}^{\mu \nu}}{2 p^{2}} \frac{d n_{F}}{d p}\right\}
$$

We add the two pieces, and go back to the full momentum variables. The final result, after adding also the antiparticle contributions, yields

$$
\begin{aligned}
\Pi_{\mathrm{m}}^{\mu \nu}(l)= & -4 m^{2} e^{2} \int \frac{d^{3} \mathbf{q}}{(2 \pi)^{3}}\left\{\frac{n_{F}}{2 q^{3}}\left[P_{\perp, \hat{\mathbf{q}}}^{\mu \nu}+\frac{\left(v_{\hat{\mathbf{q}}}^{\mu}-\tilde{v}_{\hat{\mathbf{q}}}^{\mu}\right)\left(v_{\hat{\mathbf{q}}}^{\nu}-\tilde{v}_{\hat{\mathbf{q}}}^{\nu}\right)}{2}\right]\right. \\
& \left.+\frac{1}{q^{2}} \frac{d n_{F}}{d q}\left[-\frac{P_{\perp, \hat{\mathbf{q}}}^{\mu \nu}}{2}+l_{\|, \hat{\mathbf{q}}}\left(\frac{v_{\hat{\mathbf{q}}}^{\mu} v_{\hat{\mathbf{q}}}^{\nu}}{v_{\hat{\mathbf{q}}} \cdot l}+\frac{l_{\|, \hat{\mathbf{q}}}}{2} \frac{v_{\hat{\mathbf{q}}}^{\mu} v_{\hat{\mathbf{q}}}^{\nu}}{\left(v_{\hat{\mathbf{q}}} \cdot l\right)^{2}}+\frac{1}{4} \frac{v_{\hat{\mathbf{q}}}^{\mu}\left(v_{\hat{\mathbf{q}}}^{\nu}-\tilde{v}_{\hat{\mathbf{q}}}^{\nu}\right)+v_{\hat{\mathbf{q}}}^{\nu}\left(v_{\hat{\mathbf{q}}}^{\mu}-\tilde{v}_{\hat{\mathbf{q}}}^{\mu}\right)}{v_{\hat{\mathbf{q}}} \cdot l}\right)\right]\right\} .
\end{aligned}
$$

We note that the first two terms of the second line of Eq. (33) can be written as the HTL contribution, but with a coefficient proportional to $e^{2} m^{2}$ rather than the Debye mass squared $m_{D}^{2}=e^{2} T^{2} / 3$. Note also that in the tadpole diagram the pieces that are proportional to $n_{F}(q) / q^{3}$ are in principle infrared divergent. These terms have to be evaluated using a regularization. We use dimensional regularization (DR), by assuming that the system is in $d=3+2 \epsilon$ dimensions. In this case the momentum integrals become

$$
\begin{aligned}
\int \frac{d^{d} q}{(2 \pi)^{d}} \rightarrow & \frac{4}{(4 \pi)^{2+\epsilon} \Gamma(1+\epsilon)} \int_{0}^{\infty} d q q^{2+2 \epsilon} \\
& \times \int_{-1}^{1} d \cos \theta\left(1+\epsilon \ln \left(\sin ^{2} \theta\right)\right)
\end{aligned}
$$

where $\theta$ parametrizes an angle with respect to an external vector, and $\Gamma(z)$ stands for the Gamma function. Furthermore, in $d$ dimensions one has to change the coupling constant as $e^{2} \rightarrow e^{2} \nu^{3-d}$, where $\nu$ is a renormalization scale. 
The relevant infrared radial integral is

$\nu^{-2 \epsilon} \int_{0}^{\infty} d q q^{-1+2 \epsilon} n_{F}(q)=\frac{1}{4 \epsilon}+\frac{1}{2} \ln \left(\frac{\pi T e^{-\gamma_{E}}}{2 \nu}\right)+\mathcal{O}(\epsilon)$,

where $\gamma_{E}$ is Euler's constant. However, when carrying out the angular integrals in $d=3+2 \epsilon$ dimensions, the pole term and logarithm exactly cancel, as the angular integral turns out to be proportional to $\epsilon$ (that is, it would cancel if $d=3$ ). If $d \Omega_{d}$ is the solid angle element in $d$ dimensions, and $S_{d}=2 \pi^{d} / \Gamma(d / 2)$ is the area of a $d$-dimensional unit sphere, one can check

$$
S_{3+2 \epsilon}^{-1} \int d \Omega_{3+2 \epsilon}\left(-\delta^{i j}+3 \hat{\mathbf{q}}^{i} \hat{\mathbf{q}}^{j}\right)=-\frac{2}{3} \epsilon+\mathcal{O}\left(\epsilon^{2}\right) .
$$

Thus, combing the two results one gets

$$
-4 m^{2} e^{2} \nu^{-2 \epsilon} \int \frac{d^{d} q}{(2 \pi)^{d}} \frac{n_{F}}{2 q^{3}}\left[P_{\perp, \hat{\mathbf{q}}}^{\mu \nu}+\frac{\left(v_{\hat{\mathbf{q}}}^{\mu}-\tilde{v}_{\hat{\mathbf{q}}}^{\mu}\right)\left(v_{\hat{\mathbf{q}}}^{\nu}-\tilde{v}_{\hat{\mathbf{q}}}^{\nu}\right)}{2}\right]=\frac{m^{2} e^{2}}{6 \pi^{2}} \delta^{i j}+\mathcal{O}(\epsilon),
$$

and there is no infrared divergence, but only a finite term. This finite term is ultimately needed to preserve the Ward identity obeyed by the polarization tensor, as can be checked after computing

$$
l_{\mu} \Pi_{\mathrm{m}}^{\mu \nu}(l)=4 m^{2} e^{2} \int \frac{d^{3} \mathbf{q}}{(2 \pi)^{3}} \frac{1}{q^{2}} \frac{d n_{F}}{d q}\left[\frac{l_{\|, \hat{\mathbf{q}}}^{2}}{2} \frac{v_{\hat{\mathbf{q}}}^{\nu}}{v_{\hat{\mathbf{q}}} \cdot l}-\frac{2 l_{\|, \hat{\mathbf{q}}}^{2}}{4} \frac{v_{\hat{\mathbf{q}}}^{\nu}}{v_{\hat{\mathbf{q}}} \cdot l}+\frac{l_{\|, \hat{\mathbf{q}}}}{4}\left(v_{\hat{\mathbf{q}}}^{\nu}-\tilde{v}_{\hat{\mathbf{q}}}^{\nu}\right)\right]-\frac{m^{2} e^{2}}{6 \pi^{2}} l^{j} \delta^{\nu j}=0 .
$$

Note that if we had used a cutoff regularization of the integrals, the IR divergent terms would also vanish, but the above integral would not yield the finite contribution, the last term of Eq. (38), needed to respect the gauge invariance of the computation.

We define the longitudinal and transverse parts of the photon polarization tensor in $d$ dimensions by

$$
\begin{aligned}
& \Pi^{L}\left(l_{0}, \boldsymbol{l}\right) \equiv \Pi^{00}\left(l_{0}, \boldsymbol{l}\right), \\
& \Pi^{T}\left(l_{0}, \boldsymbol{l}\right) \equiv \frac{1}{d-1}\left(\delta^{i j}-\frac{l^{i} l^{j}}{\boldsymbol{l}^{2}}\right) \Pi^{i j}\left(l_{0}, \boldsymbol{l}\right) .
\end{aligned}
$$

We then find the following mass corrections to the longitudinal and transverse parts of the polarization tensor

$$
\begin{gathered}
\Pi_{\mathrm{m}}^{\mathrm{L}}\left(l_{0}, \boldsymbol{l}\right)=\frac{e^{2} m^{2}}{2 \pi^{2}} \frac{\boldsymbol{l}^{2}}{l_{0}^{2}-\boldsymbol{l}^{2}}, \\
\Pi_{\mathrm{m}}^{\mathrm{T}}\left(l_{0}, \boldsymbol{l}\right)=\frac{e^{2} m^{2}}{2 \pi^{2}} \frac{l_{0}}{2|\boldsymbol{l}|} \ln \left(\frac{l_{0}+|\boldsymbol{l}|}{l_{0}-|\boldsymbol{l}|}\right) .
\end{gathered}
$$

Let us finally stress that Eqs. (40)-(41) remain also valid in the presence of a finite chemical potential $\mu$. In the presence of a chemical potential the particle and antiparticle contributions differ, but the final result can be recovered from Eq. (33), simply by replacing in Eq. (33)

$$
n_{F}(q) \rightarrow \frac{1}{2}\left[n_{F}(q-\mu)+n_{F}(q+\mu)\right] .
$$

After an explicit evaluation of the corresponding integrals, one reaches to the same mass corrections to the polarization tensor which are valid at high temperature. In particular, our results still hold if we take $T=0$ and keep the chemical potential $\mu$ as the high scale of the problem.

\section{COMPUTATION OF THE PHOTON POLARIZATION TENSOR FROM KINETIC THEORY}

We compute in this section the mass corrections to the photon polarization tensor as computed from kinetic theory. We use the transport approach derived from OSEFT, and focus on the vectorial component of the Wigner function. From Ref. [9], the transport equation associated to a fermion with chirality $\chi$ up to second order in the energy expansion reads

$$
\begin{aligned}
& {\left[v_{\chi}^{\mu}-\frac{e}{2 E_{q}^{2}} S_{\chi}^{\mu \nu} F_{\nu \rho}(X)\left(2 u^{\rho}-v_{\chi}^{\rho}\right)\right]} \\
& \quad \times\left(\partial_{\mu}^{X}-e F_{\mu \rho}(X) \partial_{q}^{\rho}\right) G^{\chi}(X, q)=0,
\end{aligned}
$$

where $v_{\chi}^{\mu}=q^{\mu} / E_{q}$, and we take the frame vector that defines the system as $u^{\mu}=(1, \mathbf{0})$. Furthermore

$$
G^{\chi}(X, q)=2 \pi \delta\left(Q_{m}^{\chi}\right) n^{\chi}(X, q)
$$

where $n^{\chi}(X, q)$ is the distribution function, and the delta gives the on-shell constraint, $Q_{m}^{\chi}$ being a function of the momentum and the mass. The particle contribution to the electromagnetic current is expressed, at $n=2$ order as 


$$
j^{\mu}(X)=e \sum_{\chi= \pm} \int \frac{d^{4} q}{(2 \pi)^{4}}\left[v_{\chi}^{\mu}-\frac{S_{\chi}^{\mu \nu} \Delta_{\nu}}{E_{q}}-\frac{e}{2} \frac{S_{\chi}^{\mu \nu}}{E_{q}^{2}} F_{\nu \rho}(X)\left(2 u^{\rho}-v_{\chi}^{\rho}\right)\right] 2 G^{\chi}(X, q)+\mathcal{O}\left(\frac{1}{E_{q}^{3}}\right)
$$

We ignore in this manuscript the possible effect of the spin coherence function discussed in Ref. [9], which represent coherent quantum states of mixed chiralities. We also will ignore the terms in the transport equation, on-shell constraint, and in the vector current proportional to the spin tensor $S_{\chi}^{\mu \nu}$, as they are irrelevant if the chiral chemical potential is zero, as the contribution of the two fermion chiralities makes these pieces to cancel in the macroscopic current. Those terms are relevant, though, to derive the chiral magnetic effect, which is not our goal here (see appendix of Ref. [7] for that derivation). We thus write the on-shell constraint to the considered order of accuracy

$$
q_{0}=E_{q}=q+\frac{m^{2}}{2 q} \text {. }
$$

Where $q=|\mathbf{q}|$. We now assume to be close to thermal equilibrium, such that

$$
G^{\chi}=G_{(0)}^{\chi}+\delta G^{\chi}+\cdots
$$

where $G_{(0)}^{\chi}$ is the Wigner function in thermal equilibrium. Using the transport equation, we find

$$
v^{\chi} \cdot \partial_{X} \delta G^{\chi}=e v_{\mu}^{\chi} F^{\mu \nu} \frac{\partial G_{(0)}^{\chi}}{\partial q^{\nu}}
$$

and after computing

$$
\delta j^{\mu}(X)=e \sum_{\chi= \pm} \int \frac{d^{4} q}{(2 \pi)^{4}} v_{\chi}^{\mu} \delta G^{\chi}(X, q)
$$

one derives the polarization tensor as

$$
\Pi^{\mu \nu}=\frac{\delta j^{\mu}}{\delta A_{\nu}} .
$$

It is not difficult to find the particle contribution to the polarization tensor, which reads

$$
\begin{aligned}
\Pi^{\mu \nu}(l)= & e^{2} \sum_{\chi= \pm} \int \frac{d^{3} \mathbf{q}}{(2 \pi)^{3}}\left(g^{\mu \nu}-\frac{l^{\mu} v_{m}^{\nu}+v_{m}^{\mu} l^{\nu}}{l \cdot v_{m}}+L^{2} \frac{v_{m}^{\mu} v_{m}^{\nu}}{\left(l \cdot v_{m}\right)^{2}}\right) \\
& \times \frac{n_{F}\left(q_{0}=E_{q}\right)}{E_{q}}
\end{aligned}
$$

where $L^{2}=l_{0}^{2}-l^{2}$, and

$$
v_{m}^{\mu}=v_{\hat{\mathbf{q}}}^{\mu}-\delta^{\mu i} v_{\hat{\mathbf{q}}}^{i} \frac{m^{2}}{2 q^{2}},
$$

for the particles. A similar expression holds for the antiparticles.

We compute all the pieces up to $\mathcal{O}\left(\mathrm{m}^{2}\right)$, by noting that

$$
\begin{gathered}
\frac{1}{E_{q}}=\frac{1}{q}-\frac{m^{2}}{2 q^{3}}+\cdots \\
\frac{1}{l \cdot v_{m}}=\frac{1}{l \cdot v_{\hat{\mathbf{q}}}}-\frac{\boldsymbol{l} \cdot \mathbf{v}_{\hat{\mathbf{q}}}}{\left(l \cdot v_{\hat{\mathbf{q}}}\right)^{2}} \frac{m^{2}}{2 q^{2}}+\cdots \\
n_{F}\left(E_{q}\right)=n_{F}(q)+\frac{m^{2}}{2 q} \frac{d n_{F}}{d q}+\cdots
\end{gathered}
$$

We thus find that the polarization tensor can be written as

$$
\Pi^{\mu \nu}(l)=\Pi_{\mathrm{htl}}^{\mu \nu}(l)+\Pi_{\mathrm{m}}^{\mu \nu}(l) .
$$

The HTL part, as arising from particles and antiparticles of the two possible chiralities, reads

$$
\begin{aligned}
\Pi_{\mathrm{htl}}^{\mu \nu}(l)= & 4 e^{2} \int \frac{d^{3} \mathbf{q}}{(2 \pi)^{3}}\left(g^{\mu \nu}-\frac{l^{\mu} v_{\hat{\mathbf{q}}}^{\nu}+v_{\hat{\mathbf{q}}}^{\mu} l^{\nu}}{l \cdot v_{\hat{\mathbf{q}}}}+L^{2} \frac{v_{\hat{\mathbf{q}}}^{\mu} v_{\hat{\mathbf{q}}}^{\nu}}{\left(l \cdot v_{\hat{\mathbf{q}}}\right)^{2}}\right) \\
& \times \frac{n_{F}(q)}{q} .
\end{aligned}
$$

While the leading mass correction is

$$
\begin{aligned}
\Pi_{\mathrm{m}}^{\mu \nu}(l)= & 4 e^{2} m^{2} \int \frac{d^{3} \mathbf{q}}{(2 \pi)^{3}}\left(g^{\mu \nu}-\frac{l^{\mu} v_{\hat{\mathbf{q}}}^{\nu}+v_{\hat{\mathbf{q}}}^{\mu} l^{\nu}}{l \cdot v_{\hat{\mathbf{q}}}}+L^{2} \frac{v_{\hat{\mathbf{q}}}^{\mu} v_{\hat{\mathbf{q}}}^{\nu}}{\left(l \cdot v_{\hat{\mathbf{q}}}\right)^{2}}\right)\left(\frac{1}{2 q^{2}} \frac{d n_{F}(q)}{d q}\right) \\
& +4 e^{2} m^{2} \int \frac{d^{3} \mathbf{q}}{(2 \pi)^{3}}\left(g^{\mu \nu}-l_{0} \frac{l^{\mu} v_{\hat{\mathbf{q}}}^{\nu}+v_{\hat{\mathbf{q}}}^{\mu} l^{\nu}}{\left(l \cdot v_{\hat{\mathbf{q}}}\right)^{2}}-L^{2} \frac{v_{\hat{\mathbf{q}}}^{\mu} v_{\hat{\mathbf{q}}}^{\nu}}{\left(l \cdot v_{\hat{\mathbf{q}}}\right)^{2}}+2 L^{2} l_{0} \frac{v_{\hat{\mathbf{q}}}^{\mu} v_{\hat{\mathbf{q}}}^{\nu}}{\left(l \cdot v_{\hat{\mathbf{q}}}\right)^{3}}\right. \\
& \left.-\frac{\delta^{\mu i} v_{\hat{\mathbf{q}}}^{i} l^{\nu}+\delta^{\nu i} v_{\hat{\mathbf{q}}}^{i} \hat{l}^{\mu}}{l \cdot v_{\hat{\mathbf{q}}}}+L^{2} \frac{\delta^{\mu i} v_{\hat{\mathbf{q}}}^{i} v_{\hat{\mathbf{q}}}^{\nu}+\delta^{\nu} v_{\hat{\mathbf{q}}}^{i} v_{\hat{\mathbf{q}}}^{\mu}}{\left(l \cdot v_{\hat{\mathbf{q}}}\right)^{2}}\right)\left(-\frac{n_{F}(q)}{2 q^{3}}\right) .
\end{aligned}
$$


One can check that

$$
l_{\mu} \Pi_{\mathrm{m}}^{\mu \nu}(l)=0,
$$

so that the Ward identity is respected for the mass dependent pieces of the polarization tensor at this order. Note that the first integral of Eq. (58) has the same structure than the HTL contribution, but it is proportional to the fermion mass squared. This contribution was also found out in the diagrammatic computation of Sec. III. The second integral contains IR divergencies, but are of a quite different structure as those appearing in the diagrammatic computation, see Eq. (37). The apparent IR divergencies here are clearly nonlocal. We evaluate these integrals using DR.

Note that we only need to evaluate the integral

$$
\begin{aligned}
I_{1} \equiv & \int_{-1}^{1} d \cos \theta\left(1-\cos ^{2} \theta\right)^{\epsilon} \frac{1}{l_{0}-|\boldsymbol{l}| \cos \theta} \\
= & \frac{1}{|\boldsymbol{l}|}\left\{\ln \left(\frac{l_{0}+|\boldsymbol{l}|}{l_{0}-|\boldsymbol{l}|}\right)+\epsilon\left[\ln (4) \ln \left(\frac{l_{0}+|\boldsymbol{l}|}{l_{0}-|\boldsymbol{l}|}\right)\right.\right. \\
& \left.\left.+\mathrm{Li}_{2}\left(-\frac{2|\boldsymbol{l}|}{l_{0}-|\boldsymbol{l}|}\right)-\mathrm{Li}_{2}\left(\frac{2|\boldsymbol{l}|}{l_{0}+|\boldsymbol{l}|}\right)\right]\right\}+\mathcal{O}\left(\epsilon^{2}\right),
\end{aligned}
$$

where $\mathrm{Li}_{2}$ stands for the Euler polylogarithmic function of order 2. All the remaining non-local integrals can be deduced from this one, after simple manipulations.

An explicit computation shows that after angular integration in $d=3+2 \epsilon$ dimensions the IR divergencies exactly cancel, but there are remaining finite pieces, which in this case turn out to be non-local, and that allows one to reproduce the same value of the photon polarization tensor that we found in Sec. III.

\section{DISCUSSION}

We used OSEFT to assess how a small fermion mass would affect the retarded photon polarization tensor at soft scales in a ultrarelativistic electromagnetic plasma. While it could be obvious that such corrections would be of order $m^{2} / T^{2}$, the effective field theory techniques we used allowed us their proper evaluation.

Our results could have also been derived from the expression of the full QED polarization tensor, by assuming that both the external momentum and the fermion mass are small in front of the hard loop momentum, expanding the corresponding expressions. As this expansion produces infrared divergencies, it is important to use a regularization of all the involved integrals before the expansion. We have emphasized in the whole manuscript the relevance of using a regulator that respects the gauge symmetry, as dimensional regularization, to obtain physical results. OSEFT ultimately yields the same results of this expansion, up to possible local terms. We have presented an alternative computation from transport theory so as to be sure of the absence of possible extra pieces.

Our results should be compared to both the power and two-loop corrections to the HTL tensor that have been computed in Refs. [5,17], and [18], respectively. More precisely, we will write

$$
\Pi_{I}=\Pi_{I}^{\mathrm{htl}}+\Pi_{I}^{m}+\Pi_{I}^{\text {pow.corr }}+\Pi_{I}^{2 \text { loop }}, \quad I=L, T,
$$

where $\Pi_{I}^{m}$ were displayed in Eqs. (40), (41) and

$$
\begin{aligned}
\Pi_{L}^{\mathrm{htl}}\left(l_{0}, \boldsymbol{l}\right) & =\frac{e^{2} T^{2}}{3}\left(1-\frac{l_{0}}{2|\boldsymbol{l}|} \ln \left(\frac{l_{0}+|\boldsymbol{l}|}{l_{0}-|\boldsymbol{l}|}\right)\right), \\
\Pi_{L}^{\text {pow.corr }}\left(l_{0}, \boldsymbol{l}\right) & =-\frac{e^{2}}{4 \pi^{2}}\left(\boldsymbol{l}^{2}-\frac{l_{0}^{2}}{3}\right)\left(1-\frac{l_{0}}{2|\boldsymbol{l}|} \ln \left(\frac{l_{0}+|\boldsymbol{l}|}{l_{0}-|\boldsymbol{l}|}\right)\right), \\
\Pi_{L}^{2 \text { loop }}\left(l_{0}, \boldsymbol{l}\right) & =\frac{e^{4} T^{2} L^{2}}{8 \pi^{2} \boldsymbol{l}^{2}}, \\
\Pi_{T}^{\mathrm{htl}}\left(l_{0}, \boldsymbol{l}\right) & =\frac{e^{2} T^{2}}{3} \frac{l_{0}}{4 \boldsymbol{l}^{3}}\left(2|\boldsymbol{l}| l_{0}-L^{2} \ln \left(\frac{l_{0}+|\boldsymbol{l}|}{l_{0}-|\boldsymbol{l}|}\right)\right), \\
\Pi_{T}^{\mathrm{pow} \cdot \operatorname{corr}}\left(l_{0}, \boldsymbol{l}\right) & =\frac{e^{2}}{4 \pi^{2}}\left(\frac{l_{0}^{2}}{2}+\frac{l_{0}^{4}}{6 \boldsymbol{l}^{2}}-\frac{2 \boldsymbol{l}^{2}}{3}-\frac{l_{0}^{3}}{12 \boldsymbol{l}^{3}}\left(2 \boldsymbol{l}^{2}+l_{0}^{2}-\frac{3 \boldsymbol{l}^{4}}{l_{0}^{2}}\right) \ln \left(\frac{l_{0}+|\boldsymbol{l}|}{l_{0}-|\boldsymbol{l}|}\right)\right), \\
\Pi_{T}^{\text {2loop }}\left(l_{0}, \boldsymbol{l}\right) & =-\frac{e^{4} T^{2}}{16 \pi^{2}} \frac{l_{0}}{|\boldsymbol{l} \boldsymbol{l}|} \ln \left(\frac{l_{0}+|\boldsymbol{l}|}{l_{0}-|\boldsymbol{l}|}\right) .
\end{aligned}
$$

For simplicity, $\Pi_{I}^{\text {pow.corr }}$ above is taken at the value of the renormalization scale $\nu=T e^{-\gamma_{E} / 2-1} \sqrt{\pi} / 2$ in the MS scheme. This fixes the scale of $e^{2}=e^{2}(\nu)$ in $\Pi_{I}^{\mathrm{htl}}$.
Let us recall the meaning of every term in Eq. (61). While the HTL contribution is proportional to $e^{2} T^{2}$, the results computed in this manuscript, even if they do not 
depend on the temperature, should be viewed as a a correction of order $m^{2} / T^{2}$ to the HTL. Similarly, the power corrections are of order $l^{2} / T^{2}$ respect to the HTL, while the two-loop results are corrections of order $e^{2}$. These three corrections are of the same order if $m, l \sim e T$, and should be equally considered. However, if the mass is such that $e T<m \ll T$, then the mass corrections are dominant at soft scales, $l \sim e T$.

For example, let us take the value of the photon screening mass, defined as $-m_{S}^{2}=\Pi_{L}\left(l^{0}=0, l^{2}=-m_{S}^{2}\right)$. Calculated from the value of the longitudinal part of the polarization tensor, as given in Eq. (61), results in

$$
m_{S}^{2}=\frac{e^{2} T^{2}}{3}\left(1-\frac{e^{2}}{8 \pi^{2}}-\frac{1}{2 \pi^{2}} \frac{m^{2}}{T^{2}}\right)
$$

Note that for values $m^{2} / T^{2}>\pi \alpha$, where $\alpha$ is the electromagnetic fine structure constant $\sim 1 / 137$, the mass effects give the most important corrections.

Let us finally remind the reader here that while we focused our discussion on thermal plasmas, our results and also the power corrections of Refs. $[5,17]$ remain valid in the presence of a chemical potential, or even for high $\mu$ and $T=0$. Our results can also be easily generalized to QCD, for the mass corrections to the HTL gluon polarization tensor, after taking into account some color factors, and replacing $e^{2}$ by $g^{2} / 2$ for fermions in the fundamental representation, where $g$ is the QCD coupling constant.

Our results might be useful to obtain a better evaluation of different physical observables whenever the fermions in the plasma are not strictly massless, which is a realistic condition for most of the physical scenarios where the HTL resummation techniques have been applied so far.

\section{ACKNOWLEDGMENTS}

We thank J. M. Torres-Rincon for discussions on extensive parts of this project, and S. Carignano and J. Soto for a critical reading of the manuscript. We have been supported by the Ministerio de Ciencia e Innovación (Spain) under the project No. PID2019-110165GB-I00 (MCI/AEI/FEDER, UE), as well as by the project No. 2017-SGR-247 (Catalonia). This work was also supported by the COST Action No. CA15213 THOR.
[1] R. D. Pisarski, Phys. Rev. Lett. 63, 1129 (1989).

[2] E. Braaten and R. D. Pisarski, Nucl. Phys. B337, 569 (1990).

[3] J. Ghiglieri, A. Kurkela, M. Strickland, and A. Vuorinen, Phys. Rep. 880, 1 (2020).

[4] C. Manuel and J. M. Torres-Rincon, Phys. Rev. D 90, 076007 (2014).

[5] C. Manuel, J. Soto, and S. Stetina, Phys. Rev. D 94, 025017 (2016); 96, 129901(E) (2017).

[6] C. Manuel, J. Soto, and S. Stetina, EPJ Web Conf. 137, 07014 (2017).

[7] S. Carignano, C. Manuel, and J. M. Torres-Rincon, Phys. Rev. D 98, 076005 (2018).

[8] S. Carignano, C. Manuel, and J. M. Torres-Rincon, Phys. Rev. D 102, 016003 (2020).

[9] C. Manuel and J. M. Torres-Rincon, Phys. Rev. D 103, 096022 (2021).
[10] D. K. Hong, Phys. Lett. B 473, 118 (2000).

[11] C. W. Bauer, S. Fleming, and M. E. Luke, Phys. Rev. D 63, 014006 (2000).

[12] C. W. Bauer, S. Fleming, D. Pirjol, and I. W. Stewart, Phys. Rev. D 63, 114020 (2001).

[13] J. Chay, C. Kim, and A. K. Leibovich, Phys. Rev. D 72, 014010 (2005).

[14] A. K. Leibovich, Z. Ligeti, and M. B. Wise, Phys. Lett. B 564, 231 (2003).

[15] K. C. Chou, Z. B. Su, B. L. Hao, and L. Yu, Phys. Rep. 118, 1 (1985).

[16] J. F. Nieves and P. B. Pal, Phys. Rev. D 39, 652 (1989); 40, 2148(E) (1989).

[17] S. Carignano, C. Manuel, and J. Soto, Phys. Lett. B 780, 308 (2018).

[18] S. Carignano, M. E. Carrington, and J. Soto, Phys. Lett. B 801, 135193 (2020). 